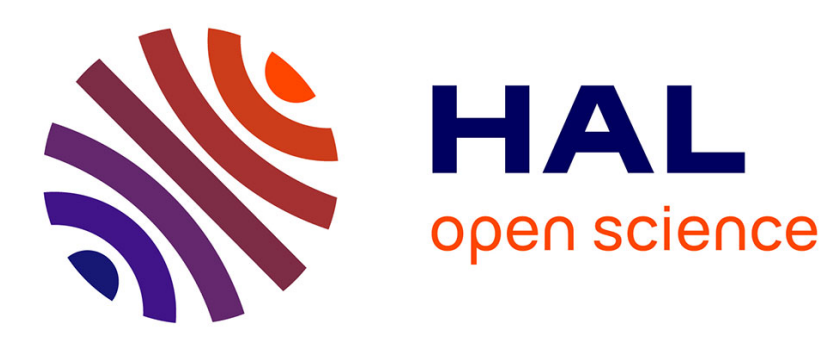

\title{
L'élection du 4 novembre 2008 et la marge de manœuvre du nouveau président américain
}

François Briatte

\section{To cite this version:}

François Briatte. L'élection du 4 novembre 2008 et la marge de manœuvre du nouveau président américain. Pouvoirs - Revue française d'études constitutionnelles et politiques, 2009, 128, pp.131-136. 10.3917/pouv.128.0129 . halshs-00338967

\section{HAL Id: halshs-00338967 \\ https://shs.hal.science/halshs-00338967}

Submitted on 14 Feb 2009

HAL is a multi-disciplinary open access archive for the deposit and dissemination of scientific research documents, whether they are published or not. The documents may come from teaching and research institutions in France or abroad, or from public or private research centers.
L'archive ouverte pluridisciplinaire HAL, est destinée au dépôt et à la diffusion de documents scientifiques de niveau recherche, publiés ou non, émanant des établissements d'enseignement et de recherche français ou étrangers, des laboratoires publics ou privés.

\section{다(1) (2)}

Distributed under a Creative Commons Attribution - ShareAlikel 4.0 International 
FRANÇOIS BRIATTE*

\section{L'ÉLECTION DU 4 NOVEMBRE 2008 ET LA MARGE DE MANOEUVRE DU NOUVEAU PRÉSIDENT AMÉRICAIN}

Le scrutin électoral du 4 novembre 2008 assure à Barack Obama la présidence des États-Unis d'Amérique, mais réformer le pays demande une compétence politique beaucoup plus importante dans un régime politique où le système de checks and balances s'applique avec une très grande efficacité.

Il est difficile de minimiser la victoire électorale de Barack Obama, que le monde entier a pu suivre grâce aux télévisions américaines - notamment CNN et MSNBC - émettant en direct sur leurs sites Internet. De nombreux sites d'analyse d'une qualité exceptionnelle, comme 270towin.com ou fivethirtyeight.com, ont immédiatement mis en perspective les résultats annoncés avec les sondages anticipés et les simulations électorales, qui donnaient Barack Obama vainqueur depuis plusieurs semaines ${ }^{1}$. Enfin, des blogs comme dailykos.com ont assuré un suivi partisan mais néanmoins quasi-professionnel de l'annonce des résultats.

* Doctorant à l'Institut d'Études Politiques de Grenoble et visiteur à la Science Studies Unit de l'Université d'Édimbourg; f.briatte@ed.ac.uk.

${ }^{1}$ Pour un aperçu des sondages pré-électoraux, cf. le site Internet pollster.com ; pour un aperçu des simulations électorales, cf. le résumé par Josep Colomer des études et modèles présentés au dernier congrès annuel de l'American Political Science Association en août 2008 : http://jcolomer.blogspot.com/2008/09/can-obama-winin-american-political.html. La simulation ayant donné la meilleure estimation du vote populaire se fondait en grande partie sur les indicateurs économiques, en accord avec le premier déterminant du vote (l'état de l'économie) donné par les 
La soirée électorale a d'abord été marquée par un taux record de participation, souvent évoqué par les médias montrant des files d'attente interminables devant les bureaux de vote. Associated Press a diffusé le 5 novembre au matin des estimations indiquant un taux de participation supérieur à $64 \%$ des électeurs potentiels, score rarement atteint au cours du XXe siècle et qui rapproche symboliquement l'événement électoral de 2008 de l'élection de John Fitzgerald Kennedy en 1960². Le premier discours prononcé par Barack Obama au cours de la nuit fait d'ailleurs allusion aux jeunes électeurs s'étant rendus aux urnes pour la première fois, et qui faisaient partie intégrante de sa stratégie électorale. Ce résultat est certainement encourageant dans une démocratie dont le système électoral est incompréhensible, voire corrompu, aux yeux d'une partie des citoyens.

La séquence des résultats État par État fut ensuite dominée par l'incertitude pendant plusieurs heures : aucun candidat ne s'est immédiatement détaché dans l'Indiana, alors que CNN annonçait dans un premier temps John McCain vainqueur en Virginie et en Floride. Une heure plus tard, Barack Obama remportait la Pennsylvanie et le New Hampshire, laissant pressentir une reproduction de la carte électorale de 2004 qui n’avait donné que 252 grands électeurs à John Kerry. Il fallut attendre encore une heure pour que Barack Obama entame un changement radical de démographie électorale en remportant l'Ohio et le Nouveau Mexique. Le

électeurs dans les sondages « sortie des urnes »; cf. Robert S. Erikson, Christopher Wlezien, « Leading Economic Indicators, the Polls and the Presidential Vote », PS : Political Science and Politics, vol. XLI, nº 4, octobre 2008, p. 703-708. ${ }^{2}$ Seth Borenstein, «Voter turnout best in generations, maybe a century », AP, 5 novembre 2008. Il s'agit des estimations de Michael McDonald, révisées à la baisse par la suite. L'estimation provisoire établie le 9 novembre se situe légèrement audessus de 61\% : http://elections.gmu.edu/preliminary_vote_2008.html. 
5 novembre, les bureaux de vote donnaient finalement Barack Obama vainqueur dans huit États ayant voté pour George Bush Jr. en $2004^{3}$. Les estimations du même jour lui assuraient d'emblée 349 grands électeurs, auxquels il faut désormais rajouter ceux de la Caroline du Nord (15 grands électeurs, précédemment acquis au parti républicain depuis 1980) et un grand électeur du Nebraska, confirmant la supériorité écrasante de sa stratégie électorale ${ }^{4}$.

Le détail des résultats électoraux, qui s'établit en comparant les sondages « sortie des urnes » (exit polls) des élections présidentielles, révèle une victoire supplémentaire en ce qui concerne les déterminants du vote populaire ${ }^{5}$. Le vote « racial » s'est ainsi exprimé de manière fondamentalement positive pour Barack Obama, avec une forte progression du vote démocrate dans l'électorat hispanique, noir et asiatique ; à l'inverse, la menace de « l'effet Bradley » (qui aurait retenu certains électeurs de sélectionner un candidat noir) ne semble pas avoir empêché la progression du vote démocrate dans l'électorat blanc par rapport à l'élection précédente. D'autres comparaisons mettent également en évidence des évolutions intéressantes : la progression du vote sur le ticket démocrate est ainsi plus marquée aux deux extrémités de l'échelle des revenus. La dimension symbolique de ces

${ }^{3}$ Pour voir ces résultats et le détail des votes, la carte électorale la plus lisible est certainement celle de $\mathrm{CNN}$ :

http://edition.cnn.com/ELECTION/2008/results/president/.

${ }^{4}$ Les résultats du Missouri n'étant pas connus au 11 novembre 2008, les estimations à cette date s'établissent à 365 grands électeurs pour le candidat démocrate.

${ }^{5}$ Le tableau livré en annexe donne quelques résultats (âge, sexe, origine ethnique, revenus et niveau de diplôme) tirés des exit polls de CNN et du New York Times pour les années 2000, 2004 et 2008. 
évolutions ne manquera pas de susciter l'intérêt des commentateurs dans les semaines à venir.

La victoire de Barack Obama est enfin une victoire des idées : le soutien apporté par l'opinion à son analyse et à ses promesses électorales sur les grands dossiers d'affaires intérieures (avec, en premier lieu, la réforme du système de santé) l'ont rapidement désigné dans les sondages comme le candidat le plus apte à conduire la politique intérieure de la nation américaine, alors que John McCain restait favori sur les thèmes du terrorisme et de la guerre en Irak. De ce point de vue, la crise financière, qui constitue de loin la « most important issue » («l'enjeu principal ») de l'élection, n'a pas vraiment révolutionné l'ensemble des préférences de l'opinion publique ${ }^{6}$, mais elle a certainement procuré un avantage décisif à Barack Obama en confortant sa position de leader d'opinion sur la conduite des affaires publiques captant le plus d'attention, comme le faisait remarquer son directeur de campagne David Axelrod dans un entretien télévisé à MSNBC. En parallèle, la nomination concomitante de Sarah Palin sur le ticket républicain a probablement permis à l'opinion publique de percevoir encore plus nettement cet « écart de compétence » déjà flagrant entre les deux camps.

Aucun de ces indicateurs préliminaires ne doit faire oublier pour autant la position institutionnelle du président américain. Le régime politique américain est un régime présidentiel doté d'un système de checks and balances très avancé, qui limite drastiquement l'étendue du pouvoir exécutif par rapport à celui des chambres

\footnotetext{
${ }^{6}$ Les sondages effectués un mois avant la faillite de Lehman Brothers désignaient déjà Barack Obama comme le candidat le plus apte à conduire la politique
} économique ; cf. les données réunies sur http://www.pollingreport.com/wh08b.htm. Sur les « most important issues », cf. les données réunies sur http://www.pollingreport.com/prioriti.htm. 
parlementaires ${ }^{7}$. En nette rupture avec son prédécesseur, Barack Obama semble également décidé à exercer un pouvoir moindre sur les nominations judiciaires, ce qui réduirait artificiellement la portée de son autorité politique. En conséquence, le sentiment de toute-puissance de la Maison Blanche est une extrapolation des réalités pratiques de l'exercice du pouvoir outre-Atlantique. Sans doute ce biais de perception est-il particulièrement fort en France, où la vie politique gravite autour de la volonté d'un pouvoir exécutif élu au suffrage universel direct et disposant d'un pouvoir de contrôle étendu sur l'agenda parlementaire. Aux États-Unis, les règles fondamentales du régime politique et le calendrier électoral assurent à l'inverse un pouvoir de décision et de veto au moins aussi important au Congrès ce que l'épisode législatif du "plan Paulson » a récemment rappelé : même en situation de crise aiguë, le président se trouve subordonné aux intérêts de la Chambre des Représentants, qui lui est rarement acquise par défaut ${ }^{8}$. Une

${ }^{7}$ Pour un aperçu du fonctionnement de la présidence américaine, cf. Charles O. Jones, The American Presidency: A Very Short Introduction, Oxford, Oxford University Press, 2007. Pour une comparaison détaillée des systèmes politiques américain et européens, cf. Yves Surel et Yves Mény, Politique comparée, Paris, Montchrestien, 2004, et en particulier les chapitres 5 et 6 sur les parlements et les exécutifs respectivement.

${ }^{8}$ Une partie de l'histoire législative du «plan Paulson » est d'ores et déjà disponible sur Wikipedia : http://en.wikipedia.org/wiki/Emergency_Economic_Stabilization_Act_of_2008. On remarquera le poids pris par l'opposition des républicains ultraconservateurs dans l'histoire du vote de ce plan, malgré leur position minoritaire au Congrès et leur affiliation partisane partagée avec la présidence de George Bush Jr. 
perspective réaliste de la vie politique américaine commence donc par une évaluation des contraintes institutionnelles qui affectent la présidence.

L'environnement politique contient naturellement d'autres données contraignantes, au premier plan desquelles l'état de l'économie. La conjoncture actuelle pèse très fortement sur l'agenda gouvernemental mais aussi sur le mode d'exercice immédiat du pouvoir présidentiel, dans la mesure où la résolution des crises financière et immobilière requiert à la fois un accord parlementaire sur les votes de crédits et un effort de coordination internationale, au moins à l'intérieur du G7/G8 ${ }^{9}$. C'est ce que rappelait Peter Baker dans le New York Times dès la publication des résultats électoraux ${ }^{10}$, et c'est aussi ce que 1'on pouvait percevoir dans l'interview donnée par Christine Lagarde à CNN en cours de soirée électorale : la ministre de l'Économie y rappelait (dans un anglais impeccable) que le prochain président américain était attendu au sommet du G20 à Washington afin de travailler sur un scenario de sortie de crise financière ${ }^{11}$.

${ }^{9}$ On lira avec intérêt le recueil d'avis d'économistes publié par voxeu.org et le Centre for Economic Policy Research à ce sujet ; cf. Barry Eichengreen et Richard Baldwin, Rescuing our Jobs and Savings: What G7/8 Leaders Can Do To Solve the Global Credit Crisis, octobre 2008 : http://www.voxeu.org/reports/crisis/crisis_141008.pdf.

${ }^{10}$ Peter Baker, «For Obama, No Time for Laurels; Now the Hard Part », New York Times, 4 novembre 2008.

${ }^{11}$ Après son élection, Barack Obama a rapidement annoncé qu'il n'interviendrait pas dans les affaires courantes pendant la période de transition (qui s'étend jusqu'à début janvier), et que par conséquent il ne se rendrait pas au G20; cf. «Obama not to attend G20 summit », AFP, 8 novembre 2008. 
Quelle est alors la marge de manœuvre effective du 44e président américain nouvellement élu ? On peut commencer par remarquer trivialement que son premier devoir consistera à ne pas reproduire les erreurs passées. Des événements comme la décision d'engager des troupes américaines en Irak à la poursuite d'hypothétiques « Weapons of Mass Destruction », ou bien la gestion du désastre intérieur causé par l'ouragan Katrina, ont clairement marqué les esprits ${ }^{12}$. Ensuite, Barack Obama possède désormais un pouvoir de nomination relativement unilatéral sur un grand nombre de postes clé dans l'administration centrale, ce qui constitue peut-être le seul moment de contrôle effectif du président sur la bureaucratie fédérale, elle-même violemment attaquée pendant la campagne par les deux candidats dans leurs références amères à «Washington and Wall Street ». Enfin, et c'est là certainement la contrainte la plus difficile à maitriser pour un président américain, il lui faudra éviter que ses projets de réforme, et notamment son universal health care plan, ne terminent leur existence dans le « cimetière législatif » que représente la Chambre des Représentants, où la majorité démocrate a également progressé ${ }^{13}$. Le folklore d'Election Day et l'investiture historique d'un

${ }^{12}$ Sur la dimension politique de l'ouragan Katrina, Wikipedia permet également de se faire une bonne idée de la controverse publique déclenchée par l'intervention du pouvoir fédéral, vivement critiquée pour son manque d'anticipation et son caractère désorganisé :

http://en.wikipedia.org/wiki/Criticism_of_government_response_to_Hurricane_Kat rina.

${ }^{13}$ Pour un historique des tentatives de réforme du système de santé américain, cf. Mark A. Peterson, « The Congressional Graveyard for Health Care Reform », in James A. Morone et Lawrence R. Jacobs (dir.), Healthy, Wealthy, and Fair : Health Care and the Good Society, New York/Oxford, Oxford University Press, 
president-elect aussi symbolique que Barack Obama ne feront disparaître que temporairement les rigidités institutionnelles de la vie politique américaine ; or c'est à leur contact que le slogan de campagne du candidat démocrate démontrera sa portée réelle. Comme l'a fait remarquer Barack Obama lui-même dans son premier discours postélectoral, sa victoire dans les urnes est une condition nécessaire mais non suffisante du changement politique aux États-Unis ${ }^{14}$.

2005, p. 235-254. Sur la relation institutionnelle entre la présidence et le Congrès, cf. David Epstein et Sharyn O’Halloran, « The Institutional Face of Presidential Power: Congressional Delegation of Authority to the President», in Robert Y. Shapiro, Martha Joynt Kumar et Lawrence R. Jacobs, Presidential Power, New York, Columbia University Press, 2000, p. 311-338.

${ }^{14}$ Discours retranscrit par le New York Times : http://www.nytimes.com/2008/11/04/us/politics/04text-obama.html. 
Comparaison des exit polls 2000/2004/2008

Tableau 1. Sexe, origine ethnique et âge

\begin{tabular}{|c|c|c|c|c|c|c|c|c|}
\hline en $\%$ & $\begin{array}{l}\text { Bush } \\
2000\end{array}$ & $\begin{array}{l}\text { Bush } \\
2004\end{array}$ & $\begin{array}{r}\text { McCain } \\
2008\end{array}$ & $\begin{array}{r}\Delta \\
2008-2004\end{array}$ & $\begin{array}{l}\text { Gore } \\
2000\end{array}$ & $\begin{array}{r}\text { Kerry } \\
2004\end{array}$ & $\begin{array}{r}\text { Obama } \\
2008 \\
\end{array}$ & $\begin{array}{r}\Delta \\
2008-2004\end{array}$ \\
\hline \multicolumn{9}{|l|}{ Sexe } \\
\hline Hommes & 53 & 55 & 48 & -7 & 42 & 44 & 49 & 5 \\
\hline Femmes & 43 & 48 & 43 & -5 & 54 & 51 & 56 & 5 \\
\hline \multicolumn{9}{|l|}{$\begin{array}{l}\text { Origine } \\
\text { ethnique }\end{array}$} \\
\hline Blancs & 54 & 58 & 55 & -3 & 42 & 41 & 43 & 2 \\
\hline Hommes & 60 & 62 & 57 & -5 & 36 & 37 & 41 & 4 \\
\hline Femmes & 49 & 55 & 53 & -2 & 48 & 44 & 46 & 2 \\
\hline Noirs & 9 & 11 & 4 & -7 & 90 & 88 & 95 & 7 \\
\hline Hispaniques & 35 & 44 & 32 & -12 & 62 & 53 & 66 & 13 \\
\hline Asiatiques & 41 & 44 & 35 & -9 & 55 & 56 & 61 & 5 \\
\hline \multicolumn{9}{|l|}{ Âge } \\
\hline $18-29$ & 46 & 45 & 32 & -13 & 48 & 54 & 66 & 12 \\
\hline $30-44$ & 49 & 53 & 46 & -7 & 48 & 46 & 52 & 6 \\
\hline $45-59$ & 49 & 51 & 49 & -2 & 48 & 48 & 49 & 1 \\
\hline $60+$ & 47 & 54 & 51 & -3 & 51 & 46 & 47 & 1 \\
\hline
\end{tabular}

Sources: CNN, The New York Times, 2008. 
Tableau 2. Niveau de revenu et niveau de diplôme

\begin{tabular}{rrrrrrrrr}
\hline & Bush & Bush & McCain & $\Delta$ & Gore & Kerry & Obama & $\Delta$ \\
en $\%$ & 2000 & 2004 & 2008 & $2008-2004$ & 2000 & 2004 & 2008 & $2008-2004$ \\
\hline
\end{tabular}

\section{Revenu}

$<\$ 15,000$

$\$ 15-30,000$

$\$ 30-50,000$

41

48

42

25

$\$ 50-75,000$

51

49

37

$\$ 75-100,000$

$\$ 100-150,000$

52

56

43

49

48

$\$ 150-200,000$

55

52
$-\quad 57$

51

$>\$ 200,000$

$-58$

50

46

-11
-5
-6
-7
-7
-6
-8
-17

57

54

49

46

45

$-$

$-$

$-$

35

$-14$

59

50

63

13

Éducation

secondaire

Université non

$\begin{array}{lll}49 & 52 & 46\end{array}$

$-6$

48

47

52

5

diplômé

51

54

47

$-7$

45

46

51

5

diplômé

Troisième

$51 \quad 52 \quad 48$

48

$-4 \quad 45$

46

50

4

cycle

$\begin{array}{lll}44 & 44 & 40\end{array}$

$-4 \quad 52$

55

58

3

Sources: CNN, The New York Times, 2008. 